\title{
Fluorescence Quenching of Xanthene Dyes during Amide Bond Formation using DMTMM
}

\author{
Kristen Patterson $\uparrow$, Misael Romero-Reyes $\dagger+$, Jennifer Heemstra $\uparrow *$ \\ $†$ Department of Chemistry, Emory University, Atlanta, Georgia 30322, United States \\ \$Department of Chemistry, Hanover College, Hanover, IN, 47243, United States
}

\begin{abstract}
Fluorophore bioconjugation to proteins, nucleic acids, and other important molecules can provide a powerful approach to sensing, imaging, and quantifying chemical and biological processes. One of the most prevalent methods for fluorophore attachment is through the formation of amide bonds, which are often facilitated by coupling agents to activate carboxylic acid moieties for subsequent nucleophilic attack by amines. DMTMM is among the most popular of these coupling agents for bioconjugation due to its ability to facilitate amide bond formation in water. After observing quenching of 5fluoresceinamine (FAM)-conjugated oligonucleotides in the presence of DMTMM, we sought to evaluate the magnitude and scope of this challenge by surveying the effect of DMTMM on a range of fluorescent dyes. A higher quenching effect was consistently observed for xanthene dyes compared to cyanine dyes. Further analysis of the impact of DMTMM on FAM shows that quenching occurs independently of whether the dye is free in solution or attached to a DNA oligonucleotide or antibody. Furthermore, we found that FAM-conjugated DNA was unable to recover its fluorescence after the removal of DMTMM and UV-Vis and NMR analysis suggests the formation of new products. However, further studies are needed to elucidate the specific analogues being formed. This research serves as a word of caution to those utilizing xanthene-containing fluorophores in bioconjugation reactions involving

DMTMM.
\end{abstract}

Amines are ubiquitous in nature and thus offer a convenient handle for bioconjugation. Consequently, amide bond formation is an essential reaction used to functionalize a wide range of molecules including drugs, proteins, antibodies, and aptamers. Generating amide bonds typically involves the conjugation of an amine and a carboxylic acid, and necessitates the release of water, making it an inherently difficult reaction to conduct under aqueous conditions. There are few coupling agents compatible with these conditions, and of these, the organic triazine derivative 4-(4,6-dimethoxy1,3,5-triazin-2-yl)-4-methyl-morpholinium chloride), or DMTMM, has emerged as a highly favorable option (Scheme S1).1 DMTMM shows comparable performance to coupling agents such as N-hydroxysulfosuccinimide (sulfo-NHS) and hexafluorophosphate azabenzotriazole tetramethyl uronium (HATU) in organic solvents and has become highly desirable for its ability to facilitate coupling reactions in aqueous environments under atmospheric conditions. ${ }^{2,3}$ While bioconjugation can be used for attachment of a wide range of molecules, coupling with fluorophores is highly common in chemical biology as this supports numerous applications including biomolecule quantification, real-time imaging and tracking, and sensing of conformation changes or the presence of analytes. ${ }^{4,5,6,7}$ This makes the selection of a well-tuned fluorophore and coupling strategy crucial to experimental design.
While exploring amide bond formation to immobilize fluorophore-labeled aptamers on a solid support, DMTMM was observed to quench the fluorescence of FAM. ${ }^{8}$ This was a significant challenge, as we had aimed to use fluorescence intensity to quantify aptamer attachment, and we recognized that this effect warranted further investigation. We subsequently exposed FAM to various concentrations of DMTMM over a period of 24 hours to determine the degree to which fluorescence intensity was affected by the presence of the coupling agent. After observing a consistent trend of fluorescence, we sought to evaluate a series of commonly used fluorescent dyes to determine how widespread this challenge was, and which fluorophore structures were most susceptible to quenching in the presence of DMTMM. We also evaluated the impact of buffer and $\mathrm{pH}$ conditions on FAM quenching and determined whether the reaction between DMTMM and fluorophores was reversible. Finally, we compared quenching for FAM either free in solution or attached to an aptamer or antibody to determine whether bioconjugation context impacted quenching. Throughout these experiments, we observe a consistent and irreversible fluorescence depletion for xanthene fluorophores, whereas cyanine fluorophores are only mildly impacted. These results suggest that researchers using DMTMM for fluorophore bioconjugation should be judicious in their choice of fluorophore structure and reaction conditions. 


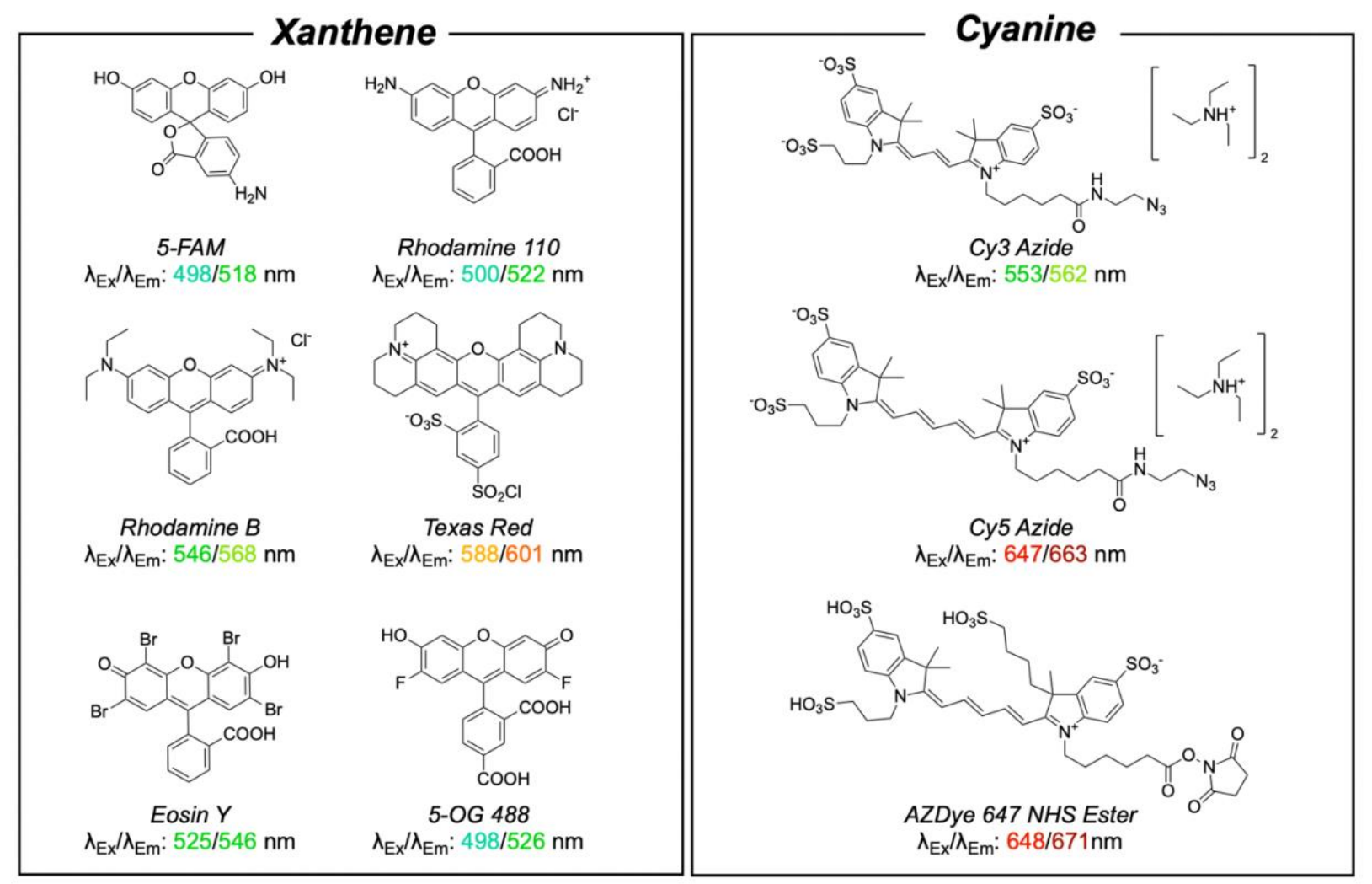

Figure 1. Chemical structures and optical properties of the xanthene and cyanine dyes investigated.

The first step of our investigation was to determine how widespread the effect of fluorescence quenching in the presence of DMTMM was. Most fluorescent dyes are based on xanthene or cyanine scaffolds, and we probed multiple examples from each structural class to elucidate relationships between dye properties and fluorescence depletion in the presence of DMTMM (Figure 1). ${ }^{9}$ To also determine the dose-dependent nature of the quenching, each dye was incubated at $1 \mu \mathrm{M}$ in the presence of varying concentrations of DMTMM ranging from $10 \mu \mathrm{M}-1 \mathrm{mM}$. A calibration curve was generated for each of the dyes, and fluorescence measurements were taken over 24 hours to observe the kinetics of depletion. To assure no photobleaching or evaporation was taking place, the 96-well plate holding the samples was covered using an opaque lid and stored in the dark. Furthermore, to minimize error, the calibration curve and samples were read on the same plate (Figure 2, Figure S1). Raw fluorescence values were compared to the calibration curve and normalized to initial fluorescence intensity.

As can be observed in Figure 2a, quenching occurred in a dose-dependent fashion, as higher concentrations of DMTMM resulted in faster reactions as well as greater overall quenching at 24 hours. However, the majority of FAM quenching was observed within the first hour of reaction. A similar general pattern of fluorescence depletion was observed across the other xanthene dyes tested, but we did observe some significant variations as a function of dye structure (Figure S2). In comparison, dyes having a cyanine core were universally more resistant to quenching after being exposed to DMTMM (Figure 2b, Figure S2). As a point of comparison, we plotted percent fluorescence depletion at 24 hours with $250 \mathrm{mM}$ DMTMM, since this represents typical conditions used for bioconjugation reactions using DMTMM (Figure 3). ${ }^{10}$ Consistent with the results described above, the dyes that suffer from the greatest quenching are all xanthenebased (Eosin Y, FAM, Rhodamine B, Oregon Green) and the cyanine dyes are among the most stable (Cy3 azide, AZDye 647, Cy5 azide). However, we also observe some dyes that defy this trend, namely Rhodamine 110 and Texas Red. While different reaction conditions and environments are necessary for different bioconjugation applications, our hope is that these data provide guidance for selecting the best fluorophores when performing amide bond formation using DMTMM.

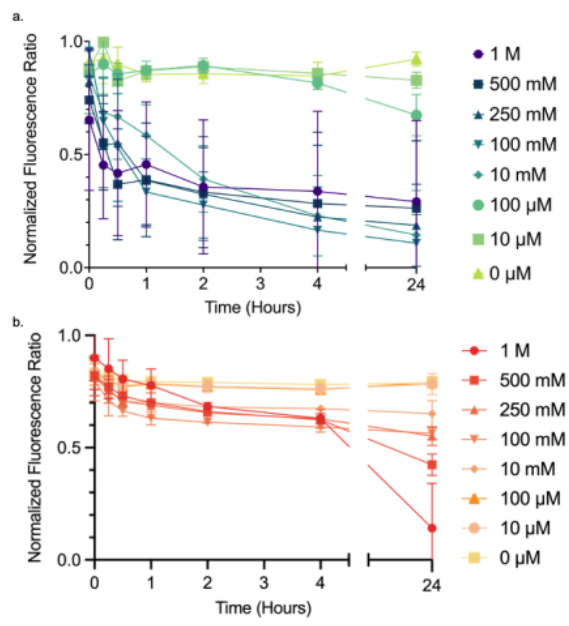

Figure 2. Fluorescence depletion of representative examples of (a) xanthene dye (FAM) and (b) cyanine dye (Cy5 azide) upon exposure to varying concentrations of 
DMTMM. All fluorescence experiments were performed at $\mathrm{N}=6$ and error bars represent standard deviation.

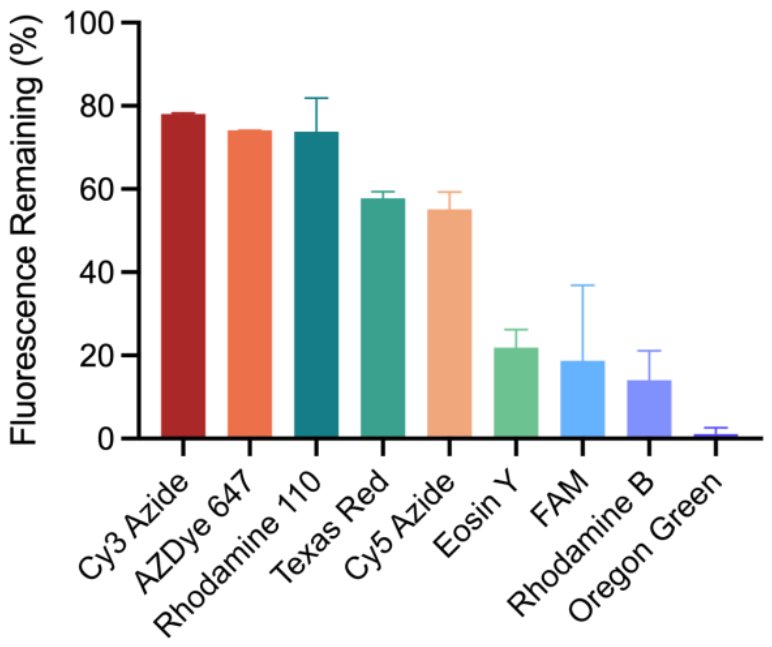

Figure 3. Percent fluorescence remaining after 24 hours of exposure to $250 \mathrm{mM}$ DMTMM. All fluorescence experiments were performed at $\mathrm{N}=6$ and error bars represent standard deviation.

In addition to evaluating the reactivity of several dyes, we were also curious to explore the effect of varying reaction conditions, including $\mathrm{pH}$ and buffer. ${ }^{11,12}$ Similar to the dye structure scan, solutions of DMTMM were prepared in a 96well plate such that dye concentration was fixed at $1 \mu \mathrm{M}$ and final DMTMM concentrations ranged from $10 \mu \mathrm{M}-1 \mathrm{mM}$. Fluorescence intensity was observed over the course of 24 hours. Across all of the buffer and $\mathrm{pH}$ conditions, the fluorescence intensity of FAM decreased with increasing concentrations of DMTMM in a similar fashion to that observed in the general dye scan (Figure S3). While significant quenching was observed under all conditions when using higher than $10 \mathrm{mM}$ DMTMM, we do observe that quenching is also faster and more pronounced at higher $\mathrm{pH}$. More importantly, however, these data show that the challenge of DMTMM quenching occurs not only in water but also in buffer systems typically used for amide bond formation.

We note that we first observed the effect of DMTMM on fluorescence when conjugating a dye-labeled aptamer to a solid support. Using the free dyes for our initial investigation of this phenomenon provided a convenient approach to rapidly test for the effect of dye structure on quenching and assess kinetics as a function of reaction conditions. However, we recognize that bioconjugation reactions are often carried out using fluorophore-labeled biomolecules such as in our example. Thus, we wanted to determine whether the impact of DMTMM on fluorescent dyes would be different when they are conjugated to biomolecules such as oligonucleotides or antibodies. We purchased the kanamycin A binding aptamer (Ky2) modified with a 5' FAM (Table S1) and monitored fluorescence quenching in MOPS buffer at $\mathrm{pH} 8$ in the presence of $300 \mathrm{mM}$ DMTMM for 24 hours. $^{13}$ A significant quenching effect was observed as FAM fluorescence was depleted by nearly $98 \%$. In a similar experimental design, a Goat Anti-Dog IgA antibody labeled with FITC was incubated in PBS buffer with $300 \mathrm{mM}$
DMTMM for 24 hours, and a similar fluorescence intensity depletion was observed (Figure 4).
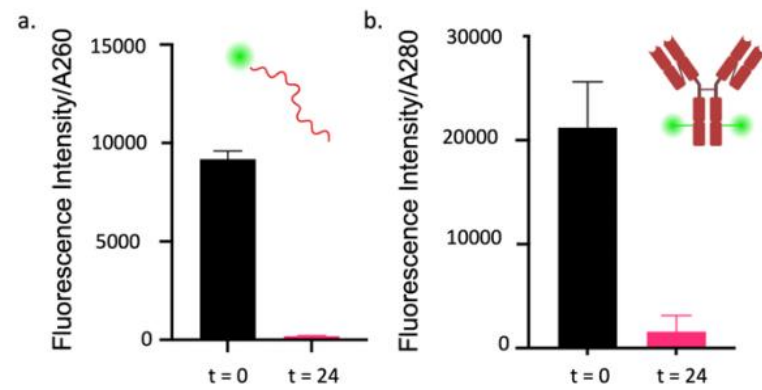

Figure 4. Fluorescence depletion of fluorescein-labeled (a) aptamer and (b) antibody. Fluorescence was monitored as a ratio to biomolecule concentration using absorbance at 260 $\mathrm{nm}$ for the aptamer and absorbance at $280 \mathrm{~nm}$ for the antibody. All fluorescence experiments were performed at $\mathrm{N}=2$ and error bars represent standard deviation.

We were then curious to determine whether this quenching effect with DMTMM was reversible. If so, then the removal of DMTMM should result in a restoration of fluorescence, and the effect of the coupling reagent on bioconjugation reactions would be a somewhat less significant issue. To probe this question, a solution of FAM-labeled Ky2 aptamer was exposed to $300 \mathrm{mM}$ DMTMM in MOPS buffer at $\mathrm{pH} 8$ for 24 hours. The reaction mixture was purified using a Monarch PCR and DNA Cleanup kit to remove excess DMTMM that would otherwise interfere with DNA quantification. The DNA was then resuspended in buffer and the DNA concentration was then measured by absorbance at $260 \mathrm{~nm}$. To observe whether recovery would be possible, we then monitored fluorescence for up to 24 hours after removal of DMTMM. As shown in Figure 5, the FAM-labeled aptamer was unable to recover a significant degree of fluorescence even 24 hours after DMTMM removal. This suggests that the reaction of DMTMM with FAM is irreversible and that use of this coupling reagent permanently damages fluorophore activity.

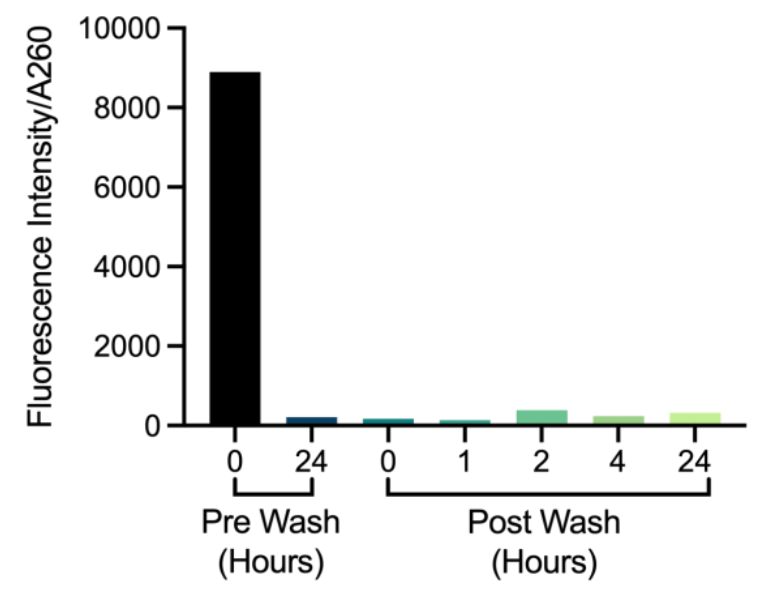

Figure 5. Probing the reversibility of the effect of DMTMM on FAM fluorescence intensity. Fluorescence decreases as expected after 24 hours after exposure to 
DMTMM and is not appreciably restored even 24 hours after DMTMM removal.

As the reaction between FAM and DMTMM was identified as being non-reversible, at least on a timescale of hours, our next step was to evaluate whether DMTMM might be covalently modifying the FAM. To study this reaction, we utilized UV-Vis and $1 \mathrm{H}$ NMR analysis. For UV-Vis analysis, a solution of $100 \mathrm{mM}$ FAM was incubated with $100 \mathrm{mM}$ DMTMM and absorbance was monitored from 200 to $800 \mathrm{~nm}$ over a 24 -hour period. We observe an initial increase in absorbance followed by a decrease and change in the ratio of peak intensity at $450 \mathrm{~nm}$, which suggests that DMTMM is undergoing chemical modification and that multiple products may be formed over time (Figure 6). ${ }^{14}$ For NMR analysis, a solution was prepared with $100 \mathrm{mM}$ FAM and $100 \mathrm{mM}$ DMTMM in deuterated DMSO and 1H NMR measurements were taken over a 24-hour period. NMR analysis showed several new peaks, suggesting a chemical reaction between the DMTMM and FAM. The reaction of the DMTMM with the carboxylic acid moiety on FAM may be one possible pathway, though it is then interesting that Rhodamine 110 is not significantly quenched despite having this same functionality. Regardless, the NMR analysis suggests that multiple products are being formed over the course of 24 hours (Figure S4-S10) and further studies would be needed to elucidate the specific analogues being formed.

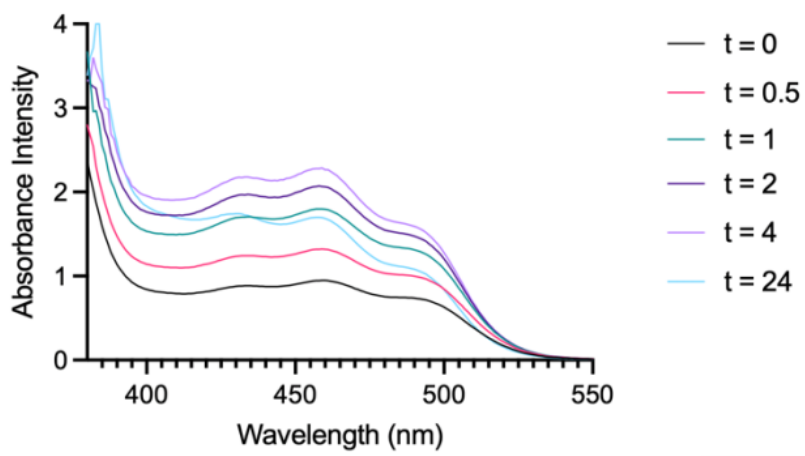

Figure 6. UV-Vis measurement of $100 \mathrm{mM}$ FAM and DMTMM solution over 24 hours.

\section{Conclusion}

In summary, we explore the impact of DMTMM on the fluorescence activity of xanthene and cyanine dyes, with a specific focus on FAM, given its widespread use in biological applications. A survey of different dye structures reveals that xanthene dyes are significantly more susceptible to fluorescence quenching by DMTMM than cyanine dyes, and we offer guidance as to the relative effect of DMTMM on the various fluorophores. We also surveyed the effect of buffer conditions and found that while all the conditions typically used for amide bond formation do give rise to significant quenching, this seems to be exacerbated at higher $\mathrm{pH}$. Furthermore, using a FAM-labeled aptamer, we demonstrate that the fluorescence depletion observed upon exposure to DMTMM is not reversible. Given the wide use of DMTMM for bioconjugation and the prevalence of xanthene fluorophores in these applications, this highlights a key challenge in bioconjugation that researchers should be aware of. In this context, $\mathrm{Cy} 3$ and other cyanine dyes present themselves as viable substitutes to xanthene dyes in bioconjugation reactions using DMTMM, especially when fluorescence intensity will be used as a means of quantification in subsequent experiments.

\section{ASSOCIATED CONTENT}

\section{Supporting Information}

The Supporting Information is available free of charge on the ACS Publications website.

\section{AUTHOR INFORMATION}

\section{Corresponding Author}

$\dagger$ Jennifer Heemstra - Department of Chemistry, Emory University, Atlanta, Georgia 30322, United States, Email: jen.heemstra@emory.edu, 0000-0002-7691-8526

\section{Author Contributions}

All authors have given approval to the final version of the manuscript.

$\dagger$ Kristen Patterson - Department of Chemistry, Emory University, Atlanta, Georgia 30322, United States, Email: kristen.patterson@emory.edu, 0000-0002-7887-3077

$\dagger$ Misael Romero-Reyes - Department of Chemistry, Hanover College, Hanover, IN, 47243, United States, Department of Chemistry, Emory University, Atlanta, Georgia 30322, United States, Email: romeroreyes@hanover.edu, 0000-0003-4614-4512

\section{Notes}

We thank Mike Hanson and the oligonucleotide and peptide synthesis facility at the University of Utah for oligonucleotide materials.

\section{ACKNOWLEDGMENT}

This work was supported by The National Science Foundation (CBET 1818476 and CHE 1904885 to J.M.H.) and the National Institutes of Health (R01GM140657 to J.M.H.)

\section{REFERENCES}

(1) Valeur, E.; Bradley, M. Amide Bond Formation: Beyond the Myth of Coupling Reagents. Chem. Soc. Rev. 2009, 38 (2), 606-631. https://doi.org/10.1039/B701677H.

(2) Kunishima, M.; Kawachi, C.; Monta, J.; Terao, K.; Iwasaki, F.; Tani, S. 4-(4,6-Dimethoxy-1,3,5-Triazin-2-Yl)-4-MethylMorpholinium Chloride: An Efficient Condensing Agent Leading to the Formation of Amides and Esters. Tetrahedron 1999, 55 (46), 13159-13170. https://doi.org/10.1016/S00404020(99)00809-1.

(3) Kamiński, Z. J.; Kolesińska, B.; Kolesińska, J.; Sabatino, G.; Chelli, M.; Rovero, P.; Błaszczyk, M.; Główka, M. L.; Papini, A. M. N-Triazinylammonium Tetrafluoroborates. A New Generation of Efficient Coupling Reagents Useful for Peptide Synthesis. J. Am. Chem. Soc. 2005, 127 (48), 16912-16920. https://doi.org/10.1021/ja054260y.

(4) Toseland, C. P. Fluorescent Labeling and Modification of Proteins. J Chem Biol 2013, 6 (3), 85-95. https://doi.org/10.1007/s12154-013-0094-5.

(5) Liu, Y.-L.; Perillo, E. P.; Ang, P.; Kim, M.; Nguyen, D. T.; Blocher, K.; Chen, Y.-A.; Liu, C.; Hassan, A. M.; Vu, H. T.; 
Chen, Y.-I.; Dunn, A. K.; Yeh, H.-C. Three-Dimensional TwoColor Dual-Particle Tracking Microscope for Monitoring DNA Conformational Changes and Nanoparticle Landings on Live Cells. ACS Nano 2020, 14 (7), 7927-7939. https://doi.org/10.1021/acsnano.9b08045.

(6) Song, H.-O.; Lee, B.; Bhusal, R. P.; Park, B.; Yu, K.; Chong, C.-K.; Cho, P.; Kim, S. Y.; Kim, H. S.; Park, H. Development of a Novel Fluorophore for Real-Time Biomonitoring System. PLOS ONE 2012, 7 (11), e48459. https://doi.org/10.1371/journal.pone.0048459.

(7) Khadria, A. S.; Senes, A. Fluorophores, Environments, and Quantification Techniques in the Analysis of Transmembrane Helix Interaction Using FRET. Biopolymers 2015, 104 (4), 247-264. https://doi.org/10.1002/bip.22667.

(8) Romero-Reyes, M. A.; Heemstra, J. M. Sequestration and Removal of Multiple Small-Molecule Contaminants Using an Optimized Aptamer-Based Ultrafiltration System. Bioconjugate Chemistry 2021. https://doi.org/10.1021/acs.bioconjchem.1c00344.

(9) Astakhova, I. K.; Wengel, J. Interfacing Click Chemistry with Automated Oligonucleotide Synthesis for the Preparation of Fluorescent DNA Probes Containing Internal Xanthene and Cyanine Dyes. Chemistry - A European Journal 2013, 19 (3), 1112-1122. https://doi.org/10.1002/chem.201202621.

(10) Li, Y.; Gabriele, E.; Samain, F.; Favalli, N.; Sladojevich, F.; Scheuermann, J.; Neri, D. Optimized Reaction Conditions for
Amide Bond Formation in DNA-Encoded Combinatorial Libraries. ACS Combinatorial Science 2016, 18. https://doi.org/10.1021/acscombsci.6b00058.

(11) Labre, F.; Mathieu, S.; Chaud, P.; Morvan, P.-Y.; Vallée, R.; Helbert, W.; Fort, S. DMTMM-Mediated Amidation of Alginate Oligosaccharides Aimed at Modulating Their Interaction with Proteins. Carbohydrate Polymers 2018, 184, 427-434. https://doi.org/10.1016/j.carbpol.2017.12.069.

(12) Li, Y.; Gabriele, E.; Samain, F.; Favalli, N.; Sladojevich, F.; Scheuermann, J.; Neri, D. Optimized Reaction Conditions for Amide Bond Formation in DNA-Encoded Combinatorial Libraries. ACS Comb Sci 2016, 18 (8), 438-443. https://doi.org/10.1021/acscombsci.6b00058.

(13) Kyung-Mi Song, Minseon Cho, Hunho Jo, Kyoungin Min, Sung Ho Jeon, Taisun Kim, Min Su Han, Ja Kang Ku, Changill Ban, "Gold nanoparticle-based colorimetric detection of kanamycin using a DNA aptamer". Anal. Biochem. 415 (2011) 175-181. https://doi.org/10.1016/j.ab.2011.04.007

(14) Arunkumar, K.; Anbazhagan, V.; Jhonsi, M.; Renganathan, R. Fluorescence Quenching of Xanthene Dyes by $\mathrm{TiO} 2$. Zeitschrift Fur Physikalische Chemie-international Journal of Research in Physical Chemistry \& Chemical Physics - Z PHYS CHEM 2007, 221, 941-948. https://doi.org/10.1524/zpch.2007.221.7.941. 